\title{
Structural Control in Weighted Voting Games*†
}

\author{
Anja Rey ${ }^{1}$ and Jörg Rothe ${ }^{2}$ \\ 1 Technische Universität Dortmund, 44221 Dortmund, Germany \\ anja.rey@tu-dortmund.de \\ 2 Heinrich-Heine-Universität Düsseldorf, 40225 Düsseldorf, Germany \\ rothe@cs.uni-duesseldorf.de
}

\begin{abstract}
Inspired by the study of control scenarios in elections and complementing manipulation and bribery settings in cooperative games with transferable utility, we introduce the notion of structural control in weighted voting games. We model two types of influence, adding players to and deleting players from a game, with goals such as increasing a given player's Shapley-Shubik or probabilistic Penrose-Banzhaf index in relation to the original game. We study the computational complexity of the problems of whether such structural changes can achieve the desired effect.
\end{abstract}

1998 ACM Subject Classification I.2.11 [Distributed Artificial Intelligence] Multiagent Systems, J.4 [Social and Behavioral Sciences] Economics

Keywords and phrases Algorithmic games theory, weighted voting games, structural control, power indices, computational complexity

Digital Object Identifier 10.4230/LIPIcs.MFCS.2016.80

\section{Introduction}

A major task in computational social choice $[42,12,13]$ is the complexity analysis of the question of whether a certain form of influence is possible in an election under some voting rule (see, e.g., [42, 13]). Bartholdi et al. [5] introduced and analyzed the notion of manipulation in elections, where one or more voters strategically change their true preference in order to make a distinguished candidate a winner. In a bribery scenario, on the other hand, an external agent tries to pay voters for them to change their votes such that a certain candidate becomes a winner, and the question is whether the briber can be successful within a given budget. This idea has been introduced and analyzed by Faliszewski et al. [24, 25]. In a third model, control, the chair of an election changes the structure of an election by adding, deleting, or partioning either voters or candidates, with the aim of making a distinguished candidate a winner [6]. In addition to these constructive types of control, destructive control - the problem of whether a given candidate can be prevented from being a winner - has also been introduced and studied by Hemaspaandra et al. [32]. Manipulation, bribery, and control have been studied for many voting systems, and we refer the reader to the book chapters by Baumeister and Rothe [10], Conitzer and Walsh [15], and Faliszewski and Rothe [27] for an overview of numerous related results. In a nutshell, whenever successful manipulative actions are possible, a high computational complexity may provide some protection against them, or at least against detecting whether such actions are possible or not for an election.

\footnotetext{
* A two-page extended abstract of this paper appeared in the proceedings of AAMAS 2016 and has been presented at the workshops CoopMAS 2016 and LOFT 2016, both with informal proceedings.

$\dagger$ This work was supported in part by DFG grant RO-1202/14-2.
} 
Similar ideas have been adapted to other fields, such as manipulation in preference aggregation [21] and manipulation, bribery, and control in judgement aggregation $[23,8,7]$ (see the book chapters by Endriss [22] and Baumeister et al. [9] for an overview). In algorithmic game theory, the question of influencing the outcome of a game has also been studied extensively. In particular, for weighted voting games, manipulation by merging a coalition of players to a single player, or by splitting a player into several players in order to increase a player's power, have been introduced by Elkind et al. [2]. Here, "power" refers to the notion of power indices, such as the Penrose-Banzhaf $[37,4,17]$ and the Shapley-Shubik index [43], measuring the significance of a player in a game (formal definitions will be given in Section 2). The complexity of beneficial merging, splitting, and annexation ${ }^{1}$, e.g., for the Shapley-Shubik index, have been studied by Aziz et al. [1] who show NP-hardness. Faliszewski and Hemaspaandra [26] show that the beneficial merging problem is in PP. Rey and Rothe [40] prove PP-hardness for beneficial merging and splitting. Other forms of manipulation have been studied in weighted voting games. For example, Zuckerman et al. [48] study manipulation of the quota. From an algorithmic point of view, this is different from our model: In their model, the number of players and thus the denominator in a power index (see Equations (1) and (2) in Section 2) remains the same but the same coalitions can have different success due to different quotas, whereas with structural control the number of players varies but all coalitions that remain in the game are equally successful before and after the change. Relatedly, Zick et al. [46, 47] study algorithmic properties of the quota. In dynamic weighted voting games, as presented by Elkind et al. [18], the quota is changed as well, but dynamically over time. The notion of bribery has been adapted from voting theory to a model in cooperative game theory, so-called path-disruption games [3], where an external player tries to bribe a coalition of players so as to reach a target node in a graph [41]. Another perspective of persuasion for weighted voting games has been studied by Freixas and Pons [30].

Inspired by the notion of control in elections, we consider control scenarios in weighted voting games. We define the problems of whether it is possible to change the structure of a game by either adding or deleting players in order to achieve certain goals. One could, for instance, think of a committee that needs a certain quota of votes so as to decide upon an issue. In order to increase the significance of some participant, an organizer might invite further participants or might choose a certain meeting schedule to make sure that originally existing participants are excluded. These structural changes could also be viewed as a change of the players' participation over time without malicious intentions. Goals include increasing and decreasing the power of a distinguished player, in relation to the player's power in the original game. Increasing and decreasing power in a game by adding or deleting players can be seen as analogues of, respectively, constructive and destructive control in elections by adding or deleting either candidates or voters. Moreover, if an exact number of players is to be added, it might be desirable to maintain an original player's power index (or to keep it upper-bounded or lower-bounded by the original value - we will say the index is nonincreasing or nondecreasing).

Note that power indices in weighted voting games can be used to model decision processes in legislative bodies such as the EU Commission, national parliaments, or the United Nations Security Council. For a real-world example, suppose there is a discussion on whether a new member will join the EU, or an old member will leave the EU. What impact does this change

1 While merging is an action of a manipulative coalition, annexation describes one manipulative player who takes over other players. This goes back to the bloc paradox [28]. 
Table 1 Overview of complexity results of control problems in weighted voting games with respect to the Shapley-Shubik and the probabilistic Penrose-Banzhaf index. Key: $k$ is the number of players to be added or deleted, respectively; $P I$ stands for power index (either $S S$ or $P B$ ); $S S$ (respectively, $P B$ ) indicates that these results are only known to hold for the Shapley-Shubik index (respectively, for the probabilistic Penrose-Banzhaf index); the other results each hold for both indices.

\begin{tabular}{|c|c|c|c|}
\hline \multirow{3}{*}{ Goal } & \multicolumn{3}{|c|}{ Control type } \\
\hline & \multicolumn{2}{|l|}{ Adding players } & \multirow{2}{*}{$\begin{array}{l}\text { Deleting players } \\
k=1\end{array}$} \\
\hline & $k$ fixed & $k$ given & \\
\hline Increase $P I$ & $\begin{array}{l}\text { PP-complete } \\
\text { (Thm. 9) }\end{array}$ & PP-hard (Thm. 8) & \multirow{2}{*}{$\begin{array}{l}\text { NP-hard }(S S) \\
(\text { Thm. 10) } \\
?\end{array}$} \\
\hline Nondecrease $P I$ & $\begin{array}{l}\text { PP-complete } \\
\text { (Thm. 9) }\end{array}$ & PP-hard (Thm. 8) & \\
\hline Decrease $P I$ & $\begin{array}{l}\text { PP-complete } \\
\text { (Thm. 9) }\end{array}$ & PP-hard (Thm. 8) & \multirow{2}{*}{$\begin{array}{l}\text { coNP-hard }(P B) \\
\text { (Thm. 11) } \\
\text { coNP-hard }(P B) \\
(\text { Thm. 13) }\end{array}$} \\
\hline Nonincrease $P I$ & $\begin{array}{l}\text { PP-complete } \\
\text { (Thm. 9) }\end{array}$ & PP-hard (Thm. 8) & \\
\hline Maintain $P I$ & $\begin{array}{l}\text { coNP-hard, } \\
\text { in PP (Thm. 12) }\end{array}$ & PP-hard (Thm. 8) & coNP-hard (Thm. 13) \\
\hline
\end{tabular}

have on the power of the existing, or remaining, EU members? Will they benefit from adding or deleting other members? Or, when it has been decided already that some new members will join the EU, an old member may be interested in maintaining the same power as before the new members have joined the game. We will show that all control types to be defined are possible in weighted voting games, and we will therefore analyze the computational complexity of whether control by structural changes can be exerted successfully in a given game. The complexity depends on the control type, the goal, and on whether the number of players that can be added or deleted is fixed or is given in the problem instance. Table 1 gives an overview.

\section{Preliminaries}

A cooperative game with transferable utility $\mathcal{G}=(N, v)$ consists of a set of players $N$ and a coalitional function $v: 2^{N} \rightarrow \mathbb{R}$ assigning a value to each subset of players, called a coalition. $\mathcal{G}$ is called simple if $v$ it is monotonic (i.e., $v(C) \leq v(D)$ whenever $C \subseteq D \subseteq N$ ) and if a coalition $C$ is either winning $(v(C)=1)$ or losing $(v(C)=0)$.

Power indices are a common concept to measure a player's significance in a simple game $\mathcal{G}=(N, v)$. Two popular indices are the Penrose-Banzhaf index $[37,4]$ and the Shapley-Shubik index [43]. Let $n$ be the number of players in $\mathcal{G}$ and $i \in N$. Define $i$ 's raw Penrose-Banzhaf power index in $\mathcal{G}$ by PenroseBanzhaf* $(\mathcal{G}, i)=\sum_{C \subseteq N \backslash\{i\}}(v(C \cup\{i\})-v(C))$ and $i$ 's probabilistic Penrose-Banzhaf power index in $\mathcal{G}$ (proposed by Dubey and Shapley [17]) by

$$
\operatorname{PenroseBanzhaf}(\mathcal{G}, i)=\frac{\text { PenroseBanzhaf }^{*}(\mathcal{G}, i)}{2^{n-1}} .
$$

We say that $i$ is critical for a coalition $C$ if the marginal contribution $v(C \cup\{i\})-v(C)$ of player $i$ to coalition $C$ in the definition above is 1, i.e., if $C$ is losing but, after $i$ has joined, $C \cup\{i\}$ is winning. On the other hand, $v(C \cup\{i\})-v(C)=0$ means that $i$ is not critical for $C$. Player $i$ 's raw Shapley-Shubik power index in $\mathcal{G}$ is $\operatorname{ShapleyShubik}^{*}(\mathcal{G}, i)=$ 
$\sum_{C \subseteq N \backslash\{i\}}\|C\| !(n-1-\|C\|) !(v(C \cup\{i\})-v(C))$, which is then normalized by

$\operatorname{ShapleyShubik}(\mathcal{G}, i)=\frac{\operatorname{ShapleyShubik}^{*}(\mathcal{G}, i)}{n !}$

to obtain $i$ 's Shapley-Shubik power index in $\mathcal{G}$.

Some simple games $\mathcal{G}=(N, v)$ can be compactly represented as weighted voting games $\left(w_{1}, \ldots, w_{n} ; q\right)$, where $w_{i}, 1 \leq i \leq n$, is player $i$ 's weight and $q$ is a quota, and a coalition $C \subseteq N$ wins if $\sum_{i \in C} w_{i} \geq q$ and otherwise it loses. Note that this representation is not fully expressive, i.e., there are simple games that cannot be represented by weighted voting games. For further background on cooperative game theory, see, e.g., the textbooks by Shoam and Leyton-Brown [44] and Peleg and Sudhölter [36] and, for computational aspects, the book by Chalkiadakis et al. [14] and the book chapters by Elkind et al. [19, 20].

For some background on computational complexity, see, e.g. the textbook by Papadimitriou [35]. We use the standard notions of hardness and completeness for a complexity class with respect to many-one polynomial-time reducibility. NP is the class of decision problems that can be solved in nondeterministic polynomial time, and coNP is the class of problems whose complements are in NP. PARTITION is the following well-known NP-complete problem:

\begin{tabular}{ll}
\hline PARTITION \\
\hline Given: & $\begin{array}{l}\text { A set } A=\{1, \ldots, n\} \text { and a function } a: A \rightarrow \mathbb{N} \backslash\{0\}, i \mapsto a_{i}, \text { such that } \sum_{i=1}^{n} a_{i} \text { is } \\
\text { even. }\end{array}$ \\
Question: & $\begin{array}{l}\text { Does there exist a partition into two subsets of equal weight, that is, does there } \\
\text { exist a subset } A^{\prime} \subseteq A \text { such that } \sum_{i \in A^{\prime}} a_{i}=\sum_{i \in A \backslash A^{\prime}} a_{i} \text { ? }\end{array}$ \\
\hline
\end{tabular}

SUBSETSUm is also a well-known NP-complete problem:

\begin{tabular}{ll}
\hline & \multicolumn{1}{c}{ Subsetsum } \\
\hline Given: & A set $A=\{1, \ldots, n\}$, a function $a: A \rightarrow \mathbb{N} \backslash\{0\}, i \mapsto a_{i}$, and a positive integer $q$. \\
Question: & Is there a subset $A^{\prime} \subseteq A$ such that $\sum_{i \in A^{\prime}} a_{i}=q ?$ \\
\hline
\end{tabular}

Let $\left(a_{1}, \ldots, a_{n} ; q\right)$ and $\left(a_{1}, \ldots, a_{n}\right)$ denote SubsetSum and Partition instances, respectively. A third well-known NP-complete problem that we will need is

\begin{tabular}{ll}
\hline & \multicolumn{1}{c}{ Exact Cover By 3 -Sets $(\mathrm{X} 3 \mathrm{C})$} \\
\hline Given: & A set $B=\{1, \ldots, 3 k\}, k>0$, and a collection $\mathcal{S}=\left\{S_{1}, \ldots, S_{n}\right\}$ of subsets $S_{i} \subseteq B$ \\
& with $\left\|S_{i}\right\|=3$ for $1 \leq i \leq n$. \\
Question: & Is there an exact cover of $B$ in $\mathcal{S}$, that is, is there a subcollection $\mathcal{S}^{\prime} \subseteq \mathcal{S}$ such that \\
& $\bigcup_{S \in \mathcal{S}^{\prime}} S=B$ and $S_{i} \cap S_{j}=\emptyset$, for each $S_{i}, S_{j} \in \mathcal{S}^{\prime}, i \neq j ?$ \\
\hline
\end{tabular}

We furthermore consider the function class $\# \mathrm{P}$, the class of functions that give the number of solutions of NP problems. A function is \#P-many-one-hard if there exists a polynomial-time reduction from each function in \#P; it is \#P-parsimonious-hard if there exists such a reduction from each function in $\# \mathrm{P}$ that preserves the number of solutions. If a \#P function is \#P-many-one-hard (\#P-parsimonious-hard) it is said to be \#P-many-onecomplete (\#P-parsimonious-complete). For instance, \#SUBSETSUM and \#X3C are known to be \#P-parsimonious-complete functions. \#P is closed under addition, i.e., if $f, g \in \# \mathrm{P}$ then $f+g \in \#$ P. From the literature $[38,16,26]$ we obtain the following lemma. 
- Lemma 1 ([38, 16, 26]). Computing the raw Penrose-Banzhaf index is \#P-parsimoniouscomplete. Computing the raw Shapley-Shubik index is \#P-many-one-complete.

The complexity class PP (probabilistic polynomial time) was introduced by Gill [31] via probabilistic Turing machines; equivalently, it can be defined as the class of all decision problems $X$ for which there exist a function $f \in \# \mathrm{P}$ and a polynomial $p$ such that for all instances $x: x \in X \Longleftrightarrow f(x) \geq 2^{p(|x|)-1}$. PP is considered to be a rather large complexity class, since it contains both NP and coNP (and even $\mathrm{P}_{\|}^{\mathrm{NP}}$ as shown by Beigel et al. [11]) and since it is known to be at least as hard (in terms of polynomial-time Turing reductions) as the polynomial hierarchy (i.e., $\mathrm{PH} \subseteq \mathrm{P}^{\mathrm{PP}}$ ) by Toda's theorem [45]. PP is closed under complement (which is easy to see) and, far from being trivial, it is also closed under union and intersection [29]. We make use of the following lemma by Faliszewski and Hemaspaandra [26, Lemma 2.3] in the context of comparing a player's power in weighted voting games with respect to the probabilistic Penrose-Banzhaf and the Shapley-Shubik index.

- Lemma 2 ([26]). Let $F$ be a \#P-parsimonious-complete function. Then, the problem COMPARE- $F=\{(x, y) \mid F(x)>F(y)\}$ is PP-complete.

Since \#X3C and \#SubSetSum are \#P-parsimonious-complete, Compare-\#SubSETSum and Compare-\#X3C are PP-complete. Moreover, we will use the following lemma due to Faliszewski and Hemaspaandra [26] that has been slightly adapted by Rey and Rothe [40].

- Lemma 3. Every X3C instance $\left(B^{\prime}, \mathcal{S}^{\prime}\right)$ can be transformed into an X3C instance $(B, \mathcal{S})$ where $\|B\|=3 k$ and $\|\mathcal{S}\|=n$, such that $k / n=2 / 3$ without changing the number of solutions (i.e., $\left.\# \mathrm{X} 3 \mathrm{C}(\mathrm{B}, \mathcal{S})=\# \mathrm{X} 3 \mathrm{C}\left(\mathrm{B}^{\prime}, \mathcal{S}^{\prime}\right)\right)$. Consequently, we can assume that the size of each solution in a SUBSETSUM instance is $2 n / 3$, that is, each subsequence summing up to the given quota contains the same number of elements.

We consider a restricted variant of the COMPARE-\#SUBSETSum problem, namely ComPARE-\#SubsetSum-RR as defined in [40]: Given a set $A=\{1, \ldots, n\}$ and a function $a: A \rightarrow \mathbb{N} \backslash\{0\}, i \mapsto a_{i}$, is the number of subsets of $A$ with values summing up to $(\alpha / 2)-2$, where $\alpha=\sum_{i=1}^{n} a_{i}$, greater than the number of subsets of $A$ with values summing up to $(\alpha / 2)-1$, i.e., is it true that

$$
\text { \#SubsetSum }\left(\left(a_{1}, \ldots, a_{n} ;(\alpha / 2)-2\right)\right)>\text { \#SubsetSum }\left(\left(a_{1}, \ldots, a_{n} ;(\alpha / 2)-1\right)\right) ?
$$

Let $\left(a_{1}, \ldots, a_{n}\right)$ denote an instance of COMPARE-\#SubsetSum-RR. From [40, Lemma 4.5] we obtain the following lemma.

- Lemma 4 ([40]). Compare-\#SubsetSum-RR is PP-hard.

Likewise, the analogous problem of whether $<$ holds in (3), denoted by COMPARE\#SubsetSum-ЯЯ, is PP-hard [40]. The following lemma differentiates between players that are not part of a weighted voting game and those who are but do not have any weight.

- Lemma 5. For both the probabilistic Penrose-Banzhaf index and the Shapley-Shubik index, given a weighted voting game, adding a player with weight zero does not change the original players' power indices, and the new player's power index is zero.

\section{Control Types and Goals}

We define control by adding and by deleting players in weighted voting games. For each control type, we consider goals, such as increasing or decreasing a distinguished player's 
power, in relation to the original game. We first define how adding and deleting a player affects the coalitional function for weighted voting games: For control by adding players, from a given weighted voting game $\mathcal{G}=\left(w_{1}, \ldots, w_{n} ; q\right)$ with $N=\{1, \ldots, n\}$ and a set $M=\{n+1, \ldots, n+m\}$ of $m$ unregistered players with weights $w_{n+1}, \ldots, w_{n+m}$, we obtain a new game $\mathcal{G}_{\cup M}=\left(w_{1}, \ldots, w_{n+m} ; q\right)$.

For example, we consider the following decision problem for a power index PI:

\begin{tabular}{ll}
\hline \multicolumn{1}{c}{ Control by Adding Players to Increase PI } \\
\hline Given: & $\begin{array}{l}\text { A weighted voting game } \mathcal{G} \text { with players } N=\{1, \ldots, n\} \text {, a set } M \text { of unregistered } \\
\text { players with weights } w_{n+1}, \ldots, w_{n+m}, \text { a distinguished player } p \in N, \text { and a positive } \\
\text { integer } k .\end{array}$ \\
Question: & $\begin{array}{l}\text { Can at most } k \text { players } M^{\prime} \subseteq M \text { be added to } \mathcal{G} \text { such that for the new game } \mathcal{G}_{\cup M^{\prime}} \text { it } \\
\text { holds that } \operatorname{PI}\left(\mathcal{G}_{\cup M^{\prime}}, p\right)>\operatorname{PI}(\mathcal{G}, p) ?\end{array}$ \\
\hline
\end{tabular}

Analogously, we can ask whether the game can be controlled so as to gain the opposite effect, and decrease a certain player's index. In these cases, hardness in terms of complexity can be seen as a shield to prevent a game from being controlled to improve a player's significance or to worsen a player's significance. On the other hand, we also consider the following control question: Is it possible to add players to a game without changing the distribution of power among the original players?

We can ask analogous questions with the same aims for removing players from the game. Deleting a subset $M \subseteq N$ of $m$ players from a weighted voting game $\mathcal{G}=\left(w_{1}, \ldots, w_{n} ; q\right)$ yields a weighted voting game $\mathcal{G} \backslash M=\left(w_{j_{1}}, \ldots, w_{j_{n-m}} ; q\right)$ with $\left\{j_{1}, \ldots, j_{n-m}\right\}=N \backslash M{ }^{2}$

For instance, we define the following decision problem for a power index PI:

\begin{tabular}{ll}
\hline & \multicolumn{1}{c}{ Control By Deleting Players to Increase PI } \\
\hline Given: & A weighted voting game $\mathcal{G}$ with players $N=\{1, \ldots, n\}$, a distinguished player \\
& $p \in N$, and a positive integer $k<\|N\|$. \\
Question: & Can at most $k$ players $M^{\prime} \subseteq N \backslash\{p\}$ be deleted from $\mathcal{G}$ such that in the new game \\
& $\mathcal{G} \backslash M^{\prime}$ it holds that $\operatorname{PI}\left(\mathcal{G} \backslash M^{\prime}, p\right)>\operatorname{PI}(\mathcal{G}, p) ?$ \\
\hline
\end{tabular}

Again, we can analogously define the variations of this problem where the goal is not to increase some player's power index but to decrease or to maintain it.

- Example 6. Let $\mathcal{G}=(N, v)$ be a weighted voting game with six players in $N=$ $\{1,2,3,4,5,6\}$ represented by $(1,2,2,3,4,5 ; 10)$. Let $k=1$, that is, one player can be removed from the game. Table 2 lists the players' probabilistic Penrose-Banzhaf and ShapleyShubik power indices for the resulting games. Note that fractions are sometimes expanded or reduced to a comparable denominator.

Consider the Penrose-Banzhaf index. Player 1, 4, 5 and 6 with indices of 1/8, 5/16, 3/8, and $9 / 16$, respectively, cannot improve from any other player being deleted. However, e.g., player 1's index can be decreased to $1 / 16$ when removing player 5 and is maintained in the other cases. Players 2 and 3 can benefit from the other one being removed, as the index increases from $3 / 16$ to $1 / 4$.

2 One might also think of different ways to reasonably model the new game, and we will eloborate on that in Section 6. Here, we focus on the notion just presented. 
Table 2 Power distribution in the games of Example 6.

\begin{tabular}{llrrrrrr}
\hline Player $i$ & 1 & 2 & 3 & 4 & 5 & 6 \\
\hline \hline PenroseBanzhaf $(\mathcal{G}, i)$ & $\cdot 32$ & 4 & 6 & 6 & 10 & 12 & 18 \\
\hline PenroseBanzhaf $(\mathcal{G} \backslash\{1\}, i)$ & $\cdot 32$ & & 6 & 6 & 10 & 10 & 18 \\
PenroseBanzhaf $(\mathcal{G} \backslash\{2\}, i)$ & $\cdot 32$ & 4 & & 8 & 8 & 12 & 16 \\
PenroseBanzhaf $(\mathcal{G} \backslash\{3\}, i)$ & $\cdot 32$ & 4 & 8 & & 8 & 12 & 16 \\
PenroseBanzhaf $(\mathcal{G} \backslash\{4\}, i)$ & $\cdot 32$ & 4 & 4 & 4 & & 12 & 16 \\
PenroseBanzhaf $(\mathcal{G} \backslash\{5\}, i)$ & $\cdot 32$ & 2 & 6 & 6 & 10 & & 14 \\
PenroseBanzhaf $(\mathcal{G} \backslash\{6\}, i)$ & $\cdot 32$ & 4 & 4 & 4 & 8 & 8 & \\
\hline PenroseBanzhaf $(\mathcal{G} \backslash\{1,2\}, i) \cdot 32$ & & & 8 & 8 & 8 & 16 \\
\hline \hline ShapleyShubik $(\mathcal{G}, i)$ & $\cdot 60$ & 4 & 6 & 6 & 11 & 13 & 20 \\
\hline ShapleyShubik $(\mathcal{G} \backslash\{1\}, i)$ & $\cdot 60$ & & 7 & 7 & 12 & 12 & 22 \\
ShapleyShubik $(\mathcal{G} \backslash\{2\}, i)$ & $\cdot 60$ & 5 & & 10 & 10 & 15 & 20 \\
ShapleyShubik $(\mathcal{G} \backslash\{3\}, i)$ & $\cdot 60$ & 5 & 10 & & 10 & 15 & 20 \\
ShapleyShubik $(\mathcal{G} \backslash\{4\}, i)$ & $\cdot 60$ & 5 & 5 & 5 & & 15 & 30 \\
ShapleyShubik $(\mathcal{G} \backslash\{5\}, i)$ & $\cdot 60$ & 3 & 8 & 8 & 13 & & 28 \\
ShapleyShubik $(\mathcal{G} \backslash\{6\}, i)$ & $\cdot 60$ & 6 & 6 & 6 & 21 & 21 & \\
\hline ShapleyShubik $(\mathcal{G} \backslash\{1,2\}, i)$ & $\cdot 60$ & & & 10 & 10 & 10 & 30 \\
\hline
\end{tabular}

For the Shapley-Shubik index, due to normalization over the permutations of participating players, an increase of power is expected when deleting a player. As an example, player 5 has an index of $13 / 60$ in $\mathcal{G}$ which increases to $1 / 4$ if either one of the players 2,3 , or 4 is deleted, and even to $7 / 20$ if 6 is deleted. However, players can also have a disadvantage, if a player leaves the game. For instance, player 1 loses power if 5 is deleted, 2 and 3 lose power if 4 is deleted, 4 loses power if 2 or 3 are deleted, and 5 loses power if 1 is deleted. This suggests a symmetric dependence of the players. In the same way, the power of players 2 and 3 remains the same if 6 is removed, and the other way around.

From the opposite view, consider the weighted voting game represented by $(2,3,4,5 ; 10)$, two unregistered players with weights 1 and 2 , and $k=2$ (see the bottom rows for the two indices). Note that adding them both ends up in $\mathcal{G}$. Here, the four players have probabilistic Penrose-Banzhaf indices of $1 / 4,1 / 4,1 / 4$, and $1 / 2$. The first player (with weight 2 ) can only be worse off when adding any of the two players. The player with weight 3 as well as the player with weight 5 can benefit from adding both players or only the one with weight 2 . The former keeps the same index, while the latter loses power if the player with weight 1 is added. Finally, the player with weight 4 improves in every situation when adding one or two players. The first and the fourth player (with weight 2 and 5 , respectively) cannot benefit from adding players with respect to the Shapley-Shubik index. The other two can take advantage in the same cases as for the probabilistic Penrose-Banzhaf index.

In particular, the example shows that these types of control are each possible. We therefore turn to the question of how hard it is to find out whether they can be exerted successfully in a given game. Next to goals in relation to the old game, we can also compare an index either in relation to the other players' power, or in relation to a constant number. See Section 6 for initial results for this idea. If a player $i$ is deleted from a weighted voting game, any other player $j$ gains the same amount of power that $i$ would gain if $j$ were deleted [33]. 
The changes of power indices by deletion of players are bounded as follows.

- Theorem 7. After deleting the players of a subset $M \subseteq N \backslash\{i\}$ of size $m \geq 1$ from a weighted voting game $\mathcal{G}=(N, v)$, the difference between player $i$ 's old and new

1. Penrose-Banzhaf index is at most $1-2^{-m}$ and is at least $-1+2^{-m}$;

2. Shapley-Shubik index is at most $1-(n-m+1) ! / 2 n !$ and is at least $-1+(n-m-1) ! / 2(n-2) !$.

In particular, if $m=1$ player is deleted, both the Penrose-Banzhaf index and the Shapley-Shubik index of any other player can increase by at most $1 / 2$ and will decrease by at most $-1 / 2$. These bounds are tight.

\section{Increasing or Decreasing an Index}

Similarly to control by adding or deleting voters or candidates in elections, adding and deleting players are not merely inverse operations. This is due to the fact that when adding players all original players are guaranteed to be part of the game before and after the structural change, whereas when deleting players each player except the distinguished one can be removed from the game. Hardness in terms of complexity can be seen as a shield to prevent a game from being controlled to improve or worsen a player's significance.

\subsection{Control by Adding Players}

From a computational complexity point of view, we distinguish the cases where an upper bound of new players is given as defined above and where the number of new players is fixed.

- Theorem 8. Control by adding a given number of players in order to increase (decrease) a distinguished player's probabilistic Penrose-Banzhaf or Shapley-Shubik index in a weighted voting game is PP-hard.

Proof. We show PP-hardness via the techniques used by Rey and Rothe [40], Faliszewski and Hemaspaandra [26], and Zuckerman et al. [48]. By Lemma 4, Compare-\#SubSETSum-RR is PP-hard. Reducing from this problem, we map an instance $\left(a_{1}, \ldots, a_{n}\right)$ with $\alpha=\sum_{i=1}^{n} a_{i}$ to a weighted voting game $\mathcal{G}$ represented by $\left(1, a_{1}, \ldots, a_{n} ; \alpha / 2\right)$, an unregistered player with weight $w_{n+2}=1, k=1$, and distinguished player $p=1$. There is only one possible new game obtained by adding the unregistered player to the game $\mathcal{G}_{\cup\{n+1\}}$. We show that

PenroseBanzhaf $\left(\mathcal{G}_{\cup\{n+2\}}, 1\right)-\operatorname{PenroseBanzhaf}(\mathcal{G}, 1)>0$ $\Longleftrightarrow \quad$ \#SubsetSum $\left(\left(a_{1}, \ldots, a_{n} ; \alpha / 2-2\right)\right)>$ \#SubsetSum$\left(\left(a_{1}, \ldots, a_{n} ; \alpha / 2-1\right)\right)$.

It holds that

$$
\begin{gathered}
\text { PenroseBanzhaf }\left(\mathcal{G}_{\cup\{n+1\}}, 1\right)-\text { PenroseBanzhaf }(\mathcal{G}, 1) \\
=1 / 2^{n}\left(\left\|\left\{C \subseteq\{2, \ldots, n+1\} \mid 2+\sum_{i \in C} a_{i-1} \geq \alpha / 2,1+\sum_{i \in C} a_{i-1}<\alpha / 2\right\}\right\|\right. \\
\left.-\left\|\left\{C \subseteq\{2, \ldots, n+1\} \mid 1+\sum_{i \in C} a_{i-1} \geq \alpha / 2, \sum_{i \in C} a_{i-1}<\alpha / 2\right\}\right\|\right) .
\end{gathered}
$$

If for some $C \subseteq\{2, \ldots, n+1\}$ the conditions of the set in (6) are satisfied (i.e., $\sum_{i \in C} a_{i-1}<\alpha / 2$ but $\left.1+\sum_{i \in C} a_{i-1} \geq \alpha / 2\right)$, then $\alpha / 2-1=\sum_{i \in C} a_{i-1}$, since the weights and the quota are integers. If for some $C \subseteq\{2, \ldots, n+1\}$ the conditions of the set in (5) are satisfied, then $\alpha / 2-2=\sum_{i \in C} a_{i-1}$. Therefore, the term in (4) is positive if and only if the number of solutions that sum up to $\alpha / 2-2$ is greater than $\alpha / 2-1$. Thus it is PP-hard to verify whether the Penrose-Banzhaf index of a player can be increased by adding players. 
Analogously, for the goal of decreasing an index we can reduce from the PP-hard problem Compare-\#SubsetSum-ЯЯ, which is defined in [40] by switching $\alpha / 2-2$ and $\alpha / 2-1$ in the definition of COMPARE-\#SUBSETSum-RR.

Likewise, with Lemma 3 these results can be adapted to the Shapley-Shubik index.

- Remark. An upper bound of $\mathrm{NP}^{\mathrm{PP}}$ can be established whenever the number of players to be added is given. We can guess the subset of new players to be added nondeterministically. Verifying whether the different goals are satisfied is encoded in the PP-oracle. We conjecture that this problem is complete for this class.

- Theorem 9. Control by adding a fixed number of players in order to increase (decrease) a distinguished player's probabilistic Penrose-Banzhaf or Shapley-Shubik index in a weighted voting game is $\mathrm{PP}$-complete.

Proof. Since the number of players to be added is fixed, there are polynomially many combinations to be added. Therefore, we have polynomially many comparisons of power indices. No matter which goal we consider, the comparison can be done in PP by Lemmas 1 and 2 and by the facts that \#P is closed under addition and PP is closed under complement. The problem belongs to $\mathrm{PP}$, since $\mathrm{PP}$ is closed under union.

Hardness is implied by the case of $k=1$ player to be added in the proof of Theorem 8 . By Lemma 5, this also holds for any other fixed number of players to be added.

\subsection{Control by Deleting Players}

Recall that although deleting a previously added player results in the same game, the possibility to fulfill a certain goal by adding a player is not the complement of the possiblity to fulfill the complement goal by deleting a player. Initially, we obtain the following.

- Theorem 10. Control by deleting players to increase a distinguished player's ShapleyShubik index in a weighted voting game is NP-hard (even if only one player is deleted).

Proof. We show NP-hardness by means of a reduction from SubsetSum. By Lemma 3 we can assume that the satisfying solutions all have the same size $\ell$. Let $\left(a_{1}, \ldots, a_{n} ; q\right)$ be a SubsetSum instance, consider the weighted voting game $\mathcal{G}$ represented by $\left(1, a_{1}, \ldots, a_{n}, q+\right.$ $1 ; q+1)$, and consider player 1 as our distiguished player. Let $k=1$ and let $\xi=$ \#SubsetSum $\left(\left(a_{1}, \ldots, a_{n} ; q\right)\right)$ denote the number of solutions for the SubSETSum instance. Then, for the raw Shapley-Shubik index it holds that $\xi \geq 1$ if and only if deleting some player but 1 can lead to an increase of 1 's index.

If: If $\xi=0$, ShapleyShubik ${ }^{*}(\mathcal{G}, 1)$ is and remains 0 no matter which player is deleted.

Only if: Assume that $\xi \geq 1$. Then $\operatorname{ShapleyShubik}^{*}(\mathcal{G}, 1)=\xi / 2 \cdot \ell !(n+1-\ell) !+\xi / 2 \cdot(n-$ $\ell)$ ! $(\ell+1)$ !. If player $n+2$ is deleted, player 1's new raw index is $\operatorname{ShapleyShubik}^{*}(\mathcal{G} \backslash\{n+2\}, 1)=$ $\xi \cdot \ell !(n-\ell) !$. This leads to

$$
\begin{aligned}
& \operatorname{ShapleyShubik}(\mathcal{G} \backslash\{n+2\}, 1)-\operatorname{ShapleyShubik}(\mathcal{G}, 1) \\
& =\frac{1}{(n+1) !} \cdot \xi \cdot \ell !(n-\ell) ! \cdot \frac{2}{2}-\frac{1}{(n+2) !} \cdot \frac{\xi}{2} \cdot \ell !(n-\ell) !(n+1-\ell+\ell+1) \\
& \quad=\frac{1}{(n+1) !} \cdot \frac{\xi}{2}(2-1) \ell !(n-\ell) !
\end{aligned}
$$

which is greater than 0 because $\ell$ ! and $(m-\ell)$ ! are positve. 
- Theorem 11. Control by deleting players to decrease a distinguished player's PenroseBanzhaf index in a weighted voting game is coNP-hard (even if only one player can be deleted).

Proof. We show coNP-hardness by means of a reduction from the complement of PARTITION, denoted by $\overline{\text { PARTITION }}$. Letting $\left(a_{1}, \ldots, a_{n}\right)$ be a PARTition instance with $\alpha=\sum_{i=1}^{n} a_{i}$ and $\xi=$ \#PARTITION $\left(\left(a_{1}, \ldots, a_{n}\right)\right)$, we construct the control instance consisting of $\mathcal{G}=$ $\left(1, a_{1}, \ldots, a_{n}, \alpha / 2 ; \alpha / 2+1\right), p=1$, and $k=1$. We show that $\xi=0$ if and only if there exists a player whose removal from the game causes player 1's Penrose-Banzhaf power to decrease.

Only if: Assume that $\xi=0$. Then PenroseBanzhaf* $(\mathcal{G}, 1)=1$. However, if player $n+2$ with weight $\alpha / 2$ is removed, there is no coalition left player 1 is critical for. Therefore, control in order to decrease player 1's Penrose-Banzhaf index is possible.

If: Assume that $\xi \geq 0$. Then PenroseBanzhaf* $(\mathcal{G}, 1)=\xi+1$. If player $n+2$ is deleted, PenroseBanzhaf $^{*}(\mathcal{G} \backslash\{n+2\}, 1)=\xi$ and

$\operatorname{PenroseBanzhaf}\left(\mathcal{G}_{\backslash\{n+2\}}, 1\right)-\operatorname{PenroseBanzhaf}(\mathcal{G}, 1)=\frac{\xi}{2^{n}}-\frac{\xi+1}{2^{n+1}}=\frac{\xi-1}{2^{n+1}} \geq 0$.

Note that this difference is even greater than 0 , since $\xi$ is even. If a player $j, 2 \leq j \leq n+1$, is deleted, PenroseBanzhaf ${ }^{*}(\mathcal{G} \backslash\{j\}, 1)=1+\xi / 2$ and

$$
\operatorname{PenroseBanzhaf}\left(\mathcal{G}_{\backslash\{n+2\}}, 1\right)-\operatorname{PenroseBanzhaf}(\mathcal{G}, 1)=\frac{1+\frac{\xi}{2}}{2^{n}}-\frac{\xi+1}{2^{n+1}}=\frac{1}{2^{n+1}}>0 .
$$

Consequently, a decrease of player 1's Penrose-Banzhaf index is not possible by deleting any other player than 1 .

\section{Maintaining an Index}

In addition to constructive or destructive goals, we now consider situations in which an exact number of players is to be added and the goal is to either maintain a distinguished player's power index in this new game, or at least to ensure that this player's power does not increase or decrease, compared with this player's power in the old game.

For instance, control by adding players with the goal to maintain a given player's power index PI is defined as follows. The other goals of nonincreasing or nondecreasing a given player's power by adding or deleting players can be defined analogously.

\begin{tabular}{ll}
\hline \multicolumn{1}{c}{ Control by Adding Players to Maintain PI } \\
\hline Given: & $\begin{array}{l}\text { A weighted voting game } \mathcal{G} \text { with players } N=\{1, \ldots, n\}, \text { a set } M \text { of unregistered } \\
\text { players with weights } w_{n+1}, \ldots, w_{n+m}, \text { a distinguished player } p \in N \text {, and a positive } \\
\text { integer } k .\end{array}$ \\
Question: & $\begin{array}{l}\text { Can exactly } k \text { players } M^{\prime} \subseteq M \text { be added to } \mathcal{G} \text { such that for the new game } \mathcal{G}_{\cup M^{\prime}} \text { it } \\
\text { holds that } \operatorname{PI}\left(\mathcal{G}_{\cup M^{\prime}}, p\right)=\operatorname{PI}(\mathcal{G}, p) ?\end{array}$ \\
\hline
\end{tabular}

\subsection{Control by Adding Players}

Analogously to Theorem 8, since PP is closed under complement and by an alternative reduction from the complement of COMPARE-\#SUBSETSUM-ЯЯ, control by adding a given number of players in order to maintain a distinguished player's probabilistic Penrose-Banzhaf or Shapley-Shubik index in a weighted voting game is PP-hard. Similarly, whenever the number of players to be added is given in unary, these problems are in $\mathrm{NP}^{\mathrm{PP}}$. 
- Theorem 12. Control by adding a fixed number of players to maintain a distinguished player's probabilistic Penrose-Banzhaf or Shapley-Shubik index in a weighted voting game is coNP-hard and in PP.

Proof. The upper bound holds by the same argument as in Theorem 9. We can show coNP-hardness by reducing from PARTITION. By Lemma 5 , the arguments also hold for any other fixed number of players to be added.

\subsection{Control by Deleting Players}

Note again that deleting players in order to increase a power index is not the inverse of adding players in order to nonincrease the same index.

- Theorem 13. Control by deleting a player in order to maintain a distinguished player's probabilistic Penrose-Banzhaf index in a weighted voting game is coNP-hard (even if only one player can be deleted).

Proof. Again, we show coNP-hardness by means of a reduction from $\overline{\text { PARTITION }}$. Letting $\left(a_{1}, \ldots, a_{n}\right)$ be a PARTITION instance with $\alpha=\sum_{i=1}^{n} a_{i}$, we construct the game $\mathcal{G}$ represented by $\left(1, a_{1}, \ldots, a_{n}, \alpha / 2, \alpha / 2 ; \alpha / 2+1\right)$ and consider player 1 as our distiguished player. Let $k=1$ and let $\xi=\#$ Partition $\left(\left(a_{1}, \ldots, a_{n}\right)\right)$ denote the number of solutions to the PARTition instance. Then, for the raw Penrose-Banzhaf, it holds that $\xi \geq 1$ if and only if deleting any player but 1 does not maintain the index of player 1 .

If: Assume that $\xi=0$. Then PenroseBanzhaf* $(\mathcal{G}, 1)=2$. If player $n+2$ with weight $\alpha / 2$ is deleted, the raw index of player 1 is $\operatorname{PenroseBanzhaf}^{*}\left(\mathcal{G}_{\backslash\{n+2\}}, 1\right)=1$, which results in the same probabilistic Penrose-Banzhaf index. The factor of 2 is due to the fact that the raw index is twice as significant in the new game with one player less than in the old game.

Only if: Assume that $\xi \geq 1$. Then PenroseBanzhaf* $(\mathcal{G}, 1)=\xi+2$. If player $n+2$ or $n+3$ is deleted, player 1's new raw index is $\xi+1$. This leads to a higher index since $\xi+2<2(\xi+1)$. Deleting player $j, 2 \leq j \leq n+1$, leads to a raw index of $\xi / 2+2$, which means that in comparison to the old game, player 1's index is increased: $\xi+2<2(\xi / 2+2)=\xi+4$.

Hence, the problem of whether it is possible to maintain a player's probabilistic PenroseBanzhaf index is coNP-hard.

In particular, if $\xi \geq 1$ in the above proof, then deleting any player cannot lead to a nonincrease. Therefore, it also holds that $\xi \geq 1$ if and only if deleting any player but 1 does not nonincrease the probabilistic Penrose-Banzhaf index of player 1. Therefore, we get coNP-hardness for the problem where the goal is to nonincrease the probabilistic Penrose-Banzhaf by essentially the same proof. Observe that from these constructions we cannot draw further conclusion about the complexity of structural control by deleting players for neither the Shapley-Shubik nor the probabilistic Penrose-Banzhaf index.

\section{Conclusions and Future Work}

For weighted voting games, we have studied two types of control, combined with the following variants of goals: Strictly increasing or strictly decreasing a player's power index by adding or deleting at most a given number of players as well as maintaining, nondecreasing, and nonincreasing a player's power index by adding or deleting an exact number of players. As a measure of a player's power we have analyzed the well-known Shapley-Shubik power index and the probabilistic Penrose-Banzhaf power index. If the number of players to be added is given, the problems of adding players in order to obtain a change in a player's index (or at 
least allow a change in one direction) is PP-hard. And if the number of players to be added is fixed, a corresponding PP upper bound is valid, so we have PP-completeness. In the case of deleting players, we have established NP- and coNP-hardness lower bounds, even for the case of deleting exactly one player. The complexity results are summed up in Table 1.

The complexity of some control problems is left open; for instance, interesting gaps remain, e.g., between NP-hardness and PP membership as well as PP-hardness and NPP membership, and we do not know the complexity of control by deleting players in order to nondecrease a player's index. Also, considering other measures of voter power may provide further insights into the problem of structurally controlling a game. Next to classic worst-case complexity results, it would be interesting to study approximations and average cases to understand the occurrence of computationally hard instances. Especially, it is interesting to find out how frequent the occurrence of a player gaining power by adding other players (or, likewise decreasing and deleting) is. This case would not to be expected intuitively but, as we have seen in Example 6, it is possible.

So far we have only obtained results for goals in relation to the original game. Alternatively, one might think of a situation where the goal is to increase a player's significance in comparison to the other players, which can also be achieved if players are added or deleted; the distinguished player's power index remains the same, but all remaining players' indices are distributed so that they are below this value. Besides this, we can also model a scenario where a player is required to exceed a certain constant power index, and we ask whether it is possible to control a game by adding or deleting players in order to reach this index. So far, we can tell that if the number of players to be added or deleted is $k=0$, our value is $1 / 2$, and the considered power index is the Penrose-Banzhaf index, the problem is PP-complete. This might change if $k>0$ is required. We might also study the variant of obtaining an exact value. Further, there seems to be a close connection to the notion of synergies in cooperative games (see, e.g., [39]), and it will be interesting to have a closer look at related results here.

In addition to weighted voting games, other classes of cooperative games with transferable utility might of course be affected by control scenarios as well. In each case, adding and deleting players has to be well-defined. As an example, consider general (weighted) majority games. Let $\mathcal{G}=\left(w_{1}, w_{2}, \ldots, w_{n} ; \alpha(n)\right)$ be a majority game, that is, $v(C)=1$ if $\sum_{i \in C} w_{i} \geq\lfloor\alpha(n)\rfloor+1$, and $v(C)=0$ otherwise, for each $C \subseteq N$. Now, if a player is deleted, the number of players $n$ is decremented, which changes the threshold $\alpha(n)$. The new coalitional function is computed as above. Adding a player requires a set of unregistered players given by their weights, and $n$ is increased. For (weighted) threshold games, the new coalitional function is determined similarly, with the difference that the threshold does not change. One could alternatively think of weights as a percentage, and change the weights of the remaining players proportionally. Thus the new game $\mathcal{G}_{\cup M}$ is defined differently, by normalizing the sum of weights to the original value. Similarly to majority games, players now do not make an absolute but a relative contribution to the game.

Adding and deleting players can be viewed as a change over time and analyzing to what extent this influences power indices is an interesting task for future work (previously, only changing the quota over time has been studied [18]). Other games that will be interesting to study in this context include games in which the Shapley-Shubik index is easy to compute, such as weighted graph games [16]. In such games, two indices in two games can be compared in polynomial time and, therefore, if the coalition that is added to or removed from a game is known, the possibility of control is easy to detect, rendering the problems trivial. If, on the other hand, there are several possible coalitions to be added, this problem might become interesting again. Eventually, if players correspond to an edge in a game, deleting an edge may 
be interesting in the context of Braess's paradox for noncooperative congestion games (see, e.g., [34, pp. 464-465]) where, informally, an extra fast lane might lead to congestion, whereas without this lane traffic may split up to equally slower paths. Can we find a connection to control by deleting a player in a cooperative game with transferable utility?

Acknowledgments. We thank the anonymous MFCS 2916, AAMAS 2016, CoopMAS 2016, and LOFT 2016 reviewers for many helpful comments on earlier drafts of this paper.

\section{References}

1 H. Aziz, Y. Bachrach, E. Elkind, and M. Paterson. False-name manipulations in weighted voting games. Journal of Artificial Intelligence Research, 40:57-93, 2011.

2 Y. Bachrach and E. Elkind. Divide and conquer: False-name manipulations in weighted voting games. In Proc. AAMAS'08, pages 975-982. IFAAMAS, 2008.

3 Y. Bachrach and E. Porat. Path disruption games. In Proc. AAMAS'10, pages 1123-1130. IFAAMAS, 2010.

4 J. Banzhaf III. Weighted voting doesn't work: A mathematical analysis. Rutgers Law Review, 19:317-343, 1965.

5 J. Bartholdi III, C. Tovey, and M. Trick. The computational difficulty of manipulating an election. Social Choice and Welfare, 6(3):227-241, 1989.

6 J. Bartholdi III, C. Tovey, and M. Trick. How hard is it to control an election? Mathematical Computer Modelling, 16(8/9):27-40, 1992.

7 D. Baumeister, G. Erdélyi, O. Erdélyi, and J. Rothe. Control in judgment aggregation. In Proc. STAIRS'12, pages 23-34. IOS Press, 2012.

8 D. Baumeister, G. Erdélyi, O. Erdélyi, and J. Rothe. Complexity of manipulation and bribery in judgment aggregation for uniform premise-based quota rules. Mathematical Social Sciences, 76:19-30, 2015.

9 D. Baumeister, G. Erdélyi, and J. Rothe. Judgment aggregation. In J. Rothe, editor, Economics and Computation. An Introduction to Algorithmic Game Theory, Computational Social Choice, and Fair Division, chapter 6, pages 361-391. Springer-Verlag, 2015.

10 D. Baumeister and J. Rothe. Preference aggregation by voting. In J. Rothe, editor, Economics and Computation. An Introduction to Algorithmic Game Theory, Computational Social Choice, and Fair Division, chapter 4, pages 197-325. Springer-Verlag, 2015.

11 R. Beigel, L. Hemachandra, and G. Wechsung. On the power of probabilistic polynomial time: $\mathrm{P}^{\mathrm{NP}[\log ]} \subseteq$ PP. In Proc. Structures'89, pages 225-227. IEEE Computer Society Press, 1989.

12 F. Brandt, V. Conitzer, and U. Endriss. Computational social choice. In G. Weiß, editor, Multiagent Systems, pages 213-283. MIT Press, second edition, 2013.

13 F. Brandt, V. Conitzer, U. Endriss, J. Lang, and A. Procaccia, editors. Handbook of Computational Social Choice. Cambridge University Press, 2016.

14 G. Chalkiadakis, E. Elkind, and M. Wooldridge. Computational Aspects of Cooperative Game Theory. Morgan \& Claypool, 2011.

15 V. Conitzer and T. Walsh. Barriers to manipulation in voting. In F. Brandt, V. Conitzer, U. Endriss, J. Lang, and A. Procaccia, editors, Handbook of Computational Social Choice, chapter 6, pages 127-145. Cambridge University Press, 2016.

16 X. Deng and C. Papadimitriou. On the complexity of comparative solution concepts. Mathematics of Operations Research, 19(2):257-266, 1994.

17 P. Dubey and L. Shapley. Mathematical properties of the Banzhaf power index. Mathematics of Operations Research, 4(2):99-131, 1979. 
18 E. Elkind, D. Pasechnik, and Y. Zick. Dynamic weighted voting games. In Proc. AAMAS'13, pages 515-522. IFAAMAS, 2013.

19 E. Elkind, T. Rahwan, and N. Jennings. Computational coalition formation. In G. Weiß, editor, Multiagent Systems, pages 329-380. MIT Press, second edition, 2013.

20 E. Elkind and J. Rothe. Cooperative game theory. In J. Rothe, editor, Economics and Computation. An Introduction to Algorithmic Game Theory, Computational Social Choice, and Fair Division, chapter 3, pages 135-193. Springer-Verlag, 2015.

21 U. Endriss. Sincerity and manipulation under approval voting. Theory and Decision, 74(3):335-355, 2013.

22 U. Endriss. Judgment aggregation. In F. Brandt, V. Conitzer, U. Endriss, J. Lang, and A. Procaccia, editors, Handbook of Computational Social Choice, chapter 17, pages 399-426. Cambridge University Press, 2016.

23 U. Endriss, U. Grandi, and D. Porello. Complexity of judgment aggregation. Journal of Artificial Intelligence Research, 45:481-514, 2012.

24 P. Faliszewski, E. Hemaspaandra, and L. Hemaspaandra. How hard is bribery in elections? Journal of Artificial Intelligence Research, 35:485-532, 2009.

25 P. Faliszewski, E. Hemaspaandra, L. Hemaspaandra, and J. Rothe. Llull and Copeland voting computationally resist bribery and constructive control. Journal of Artificial Intelligence Research, 35:275-341, 2009.

26 P. Faliszewski and L. Hemaspaandra. The complexity of power-index comparison. Theoretical Computer Science, 410(1):101-107, 2009.

27 P. Faliszewski and J. Rothe. Control and bribery in voting. In F. Brandt, V. Conitzer, U. Endriss, J. Lang, and A. Procaccia, editors, Handbook of Computational Social Choice, chapter 7, pages 146-168. Cambridge University Press, 2016.

28 D. Felsenthal and M. Machover. Postulates and paradoxes of relative voting power - A critical re-appraisal. Theory and Decision, 38(2):195-229, 1995.

29 L. Fortnow and N. Reingold. PP is closed under truth-table reductions. Information and Computation, 124(1):1-6, 1996.

30 J. Freixas and M. Pons. Circumstantial power: Optimal persuadable voters. European Journal of Operational Research, 186:1114-1126, 2008.

31 J. Gill. Computational complexity of probabilistic Turing machines. SIAM Journal on Computing, 6(4):675-695, 1977.

32 E. Hemaspaandra, L. Hemaspaandra, and J. Rothe. Anyone but him: The complexity of precluding an alternative. Artificial Intelligence, 171(5-6):255-285, 2007.

33 R. Myerson. Conference structures and fair allocation rules. International Journal of Game Theory, 9(3):169-182, 1980.

34 N. Nisan, T. Roughgarden, É. Tardos, and V. Vazirani. Algorithmic Game Theory. Cambridge University Press, 2007.

35 C. Papadimitriou. Computational Complexity. Addison-Wesley, second edition, 1995.

36 B. Peleg and P. Sudhölter. Introduction to the Theory of Cooperative Games. SpringerVerlag, second edition, 2007.

37 L. Penrose. The elementary statistics of majority voting. Journal of the Royal Statistical Society, 109(1):53-57, 1946.

38 K. Prasad and J. Kelly. NP-completeness of some problems concerning voting games. International Journal of Game Theory, 19(1):1-9, 1990.

39 T. Rahwan, T. Michalak, and M. Wooldridge. A measure of synergy in coalitions. Technical Report arXiv:1404.2954.v1 [cs.GT], CoRR, Apr. 2014.

40 A. Rey and J. Rothe. False-name manipulation in weighted voting games is hard for probabilistic polynomial time. Journal of Artificial Intelligence Research, 50:573-601, 2014. 
41 A. Rey, J. Rothe, and A. Marple. Path-disruption games: Bribery and a probabilistic model. Theory of Computing Systems, 2016. doi:10.1007/s00224-016-9669-1.

42 J. Rothe, editor. Economics and Computation. An Introduction to Algorithmic Game Theory, Computational Social Choice, and Fair Division. Springer-Verlag, 2015.

43 L. Shapley and M. Shubik. A method of evaluating the distribution of power in a committee system. American Political Science Review, 48(3):787-792, 1954.

44 Y. Shoham and K. Leyton-Brown. Multiagent Systems. Algorithmic, Game-Theoretic, and Logical Foundations. Cambridge University Press, 2009.

45 S. Toda. PP is as hard as the polynomial-time hierarchy. SIAM Journal on Computing, 20(5):865-877, 1991.

46 Y. Zick. On random quotas and proportional representation in weighted voting games. In Proc. IJCAI'13, pages 432-438. AAAI Press, 2013.

47 Y. Zick, A. Skopalik, and E. Elkind. The Shapley value as a function of the quota in weighted voting games. In Proc. IJCAI'11, pages 490-496. AAAI Press, 2011.

48 M. Zuckerman, P. Faliszewski, Y. Bachrach, and E. Elkind. Manipulating the quota in weighted voting games. Artificial Intelligence, 180-181:1-19, 2012. 